\title{
A CLASS OF NONLINEAR ELLIPTIC SYSTEMS WITH STEKLOV-NEUMANN NONLINEAR BOUNDARY CONDITIONS
}

\author{
JULIANO D.B. DE GODOI, OLÍMPIO H. MIYAGAKI \\ AND RODRIGO S. RODRIGUES
}

\begin{abstract}
We will study a class of nonlinear elliptic systems involving Steklov-Neumann boundary conditions. We obtain results ensuring the existence of solutions when resonance and nonresonance conditions occur. The results were obtained by using variational arguments.
\end{abstract}

1. Introduction. In this work, we will show existence results for the following class of nonlinear elliptic systems with Steklov-Neumann boundary conditions:

$$
\begin{cases}-\triangle U+C(x) U=f(x, U) & \text { in } \Omega, \\ \partial U / \partial \eta=g(x, U) & \text { on } \partial \Omega,\end{cases}
$$

where

$$
U=(u, v) \in H(\Omega) \equiv H^{1}(\Omega) \times H^{1}(\Omega), \quad \Omega \subset \mathbb{R}^{N},
$$

for $N \geq 2$, is a bounded domain with $\partial \Omega \in C^{0,1}$, and

$$
\frac{\partial}{\partial \eta} \doteq \eta \cdot \nabla
$$

is a normal derivative on $\partial \Omega$. We assume the positive definite matrix on $\mathbb{R}^{2}$ for $x \in \Omega$ almost everywhere,

$$
C(x)=\left(\begin{array}{ll}
a(x) & b(x) \\
b(x) & c(x)
\end{array}\right)
$$

2010 AMS Mathematics subject classification. Primary 35J50, Secondary 35J15, 35J57, 45C05.

Keywords and phrases. Steklov-Neumann eigenvalue, variational methods, elliptic system.

The first author was partially supported by CAPES/Brazil. The second author was partially supported by INCTMAT/MCT/Brazil and CAPES proc 2531/14-3. The second author is the corresponding author.

Received by the editors on November 7, 2014. 
with $a, b, c \in L^{p}(\Omega)$, for $p \geq N / 2$, with $N \geq 3(p>1$, when $N=2)$. The nonlinearities $f=\left(f_{1}, f_{2}\right)$ and $g=\left(g_{1}, g_{2}\right)$ satisfy the following conditions:

(H1) $F, G \in C^{1}\left(\bar{\Omega} \times \mathbb{R}^{2}, \mathbb{R}\right)$, and, for all $x \in \bar{\Omega}$,

$$
\nabla_{U} F(x, U)=f(x, U) \quad \text { and } \quad \nabla_{U} G(x, U)=g(x, U),
$$

where

$$
\nabla_{U} F \doteq\left(\frac{\partial F}{\partial u}, \frac{\partial F}{\partial v}\right)
$$

(G1) There exist constants $A_{1}^{i}, A_{2}^{i}>0$ such that

$$
\left|g_{i}(x, u, v)\right| \leq A_{1}^{i}+A_{2}^{i}[|u|+|v|]^{s},
$$

with

$$
0 \leq s<\frac{N}{N-2}, \quad i=1,2,
$$

$x \in \bar{\Omega}$ and $U=(u, v) \in \mathbb{R}^{2}$.

(F1) There exist constants $B_{1}^{i}, B_{2}^{i}>0$ such that

$$
\left|f_{i}(x, u, v)\right| \leq B_{1}^{i}+B_{2}^{i}[|u|+|v|]^{t},
$$

with

$$
0 \leq t<\frac{N+2}{N-2}, \quad i=1,2,
$$

$x \in \bar{\Omega}$ and $U=(u, v) \in \mathbb{R}^{2}$.

(LG) $G$ satisfies

$$
\lim _{|U| \rightarrow+\infty}\left[\nabla_{U} G(x, U) \cdot U-2 G(x, U)\right]=+\infty,
$$

uniformly almost everywhere $x \in \partial \Omega$.

(LF) $F$ satisfies

$$
\lim _{|U| \rightarrow+\infty}\left[\nabla_{U} F(x, U) \cdot U-2 F(x, U)\right]=+\infty,
$$

uniformly almost everywhere $x \in \Omega$.

Conditions (LG) and (LF) were used in [9]. There are many papers treating the Neumann boundary conditions, see for example, $[8,11,15,16,20]$, while there are few works involving Steklov boundary conditions, see for example, $[\mathbf{1}, \mathbf{7}, \mathbf{1 4}]$. 
In [12] (see [2] for Neumann and $[\mathbf{3}, \mathbf{4}, \mathbf{5}, \mathbf{6}]$ for Steklov), we established that the eigensystem,

$$
\begin{cases}-\triangle U+C(x) U=0 & \text { in } \Omega, \\ \partial U / \partial \eta=\mu U & \text { on } \partial \Omega,\end{cases}
$$

admits a sequence of the eigenvalues, which we will call Steklov eigenvalues, $\left(\mu_{j}\right)$, such that

$$
0<\mu_{1} \leq \mu_{2} \leq \cdots \leq \mu_{j} \leq \cdots \longrightarrow+\infty \text {, as } j \rightarrow+\infty .
$$

In addition, the dimension of the eigenspace associated to the eigenvalue $\mu_{j}$ is finite, for each $j$, by the variational characterization of the first eigenvalues $\mu_{1}$,

$$
\mu_{1}\|U\|_{2, \partial}^{2} \leq\|U\|_{C}^{2}, \quad \text { for all } U \in H(\Omega),
$$

where

$$
\|U\|_{2, \partial}^{2}=\|u\|_{2, \partial}^{2}+\|v\|_{2, \partial}^{2} \quad \text { and } \quad\|U\|_{C}^{2}=\langle U, U\rangle_{C},
$$

with the following inner product for $H(\Omega)$ :

$$
\langle U, V\rangle_{C}=\int_{\Omega}[\nabla U \cdot \nabla V+\langle C(x) U, V\rangle] d x
$$

When $C=i d$, we denote $\|U\|_{H}=\sqrt{\langle U, U\rangle}$ as the usual norm in $H(\Omega)$, which is an equivalent norm to $\|U\|_{C}$.

In our work, we also use the following notation for norms:

$$
\begin{aligned}
\|w\|_{q, \partial}^{q} & \doteq \int_{\partial \Omega}|w|^{q} d \sigma \\
\|w\|_{q}^{q} & =\int_{\Omega}|w|^{q} d x
\end{aligned}
$$

and

$$
\|U\|_{q}^{q}=\|u\|_{q}^{q}+\|v\|_{q}^{q}
$$

for $U=(u, v) \in\left[L^{q}(\Omega)\right]^{2}$ and $w \in L^{q}(\Omega)$, with $1 \leq q<\infty$.

In $[\mathbf{1 2}]$, we also prove that the eigensystem

$$
\begin{cases}-\triangle U+C(x) U=\lambda U & \text { in } \Omega \\ \partial U / \partial \eta=0 & \text { on } \partial \Omega\end{cases}
$$


admits a sequence of the eigenvalues, called Neumann eigenvalues, $\left(\lambda_{j}\right)$, such that

$$
0<\lambda_{1} \leq \lambda_{2} \leq \lambda_{3} \leq \cdots \leq \lambda_{j} \leq \cdots \longrightarrow+\infty, \quad \text { as } j \rightarrow+\infty .
$$

In addition, the dimension of the eigenspace associated to the eigenvalue $\lambda_{j}$ is finite, for each $j$, by the variational characterization of the first eigenvalues $\lambda_{1}$,

$$
\lambda_{1}\|U\|_{2}^{2} \leq\|U\|_{C}^{2}, \quad \text { for all } U \in H(\Omega) .
$$

Our results are established in accordance with the interactions of the nonlinearity on the boundary $g$, with Steklov spectrum, and the reaction term $f$, with Neumann spectrum.

By a weak solution for system (1.1), we mean:

Definition 1.1. $U \in H(\Omega)$ is a weak solution for system (1.1) when $\int_{\Omega}[\nabla U \cdot \nabla V+\langle C(x) U, V\rangle] d x=\int_{\Omega} f(x, U) \cdot V d x+\int_{\partial \Omega} g(x, U) \cdot V d \sigma$, for all $V \in H(\Omega)$.

Our first result describes when the nonlinearities are below the first eigenvalues.

Theorem 1.2. In addition to $(\mathbf{P}),(\mathbf{H 1}),(\mathbf{G 1})$ and $(\mathbf{F 1})$, suppose the following condition:

(A1) There exist constants $\lambda, \mu \in \mathbb{R}$ such that

$$
\limsup _{|U| \rightarrow+\infty} \frac{2 G(x, U)}{u^{2}+v^{2}} \leq \mu<\mu_{1}
$$

and

$$
\limsup _{|U| \rightarrow+\infty} \frac{2 F(x, U)}{u^{2}+v^{2}} \leq \lambda<\lambda_{1}
$$

uniformly for $x \in \bar{\Omega}$, with $\lambda_{1} \mu+\mu_{1} \lambda<\mu_{1} \lambda_{1}$. Then system (1.1) possesses at least one solution $U \in H(\Omega)$. 
In Figure 1, the hatched region in the Cartesian plane $\lambda \mu$ illustrates $\lambda_{1} \mu+\mu_{1} \lambda<\mu_{1} \lambda_{1}$.

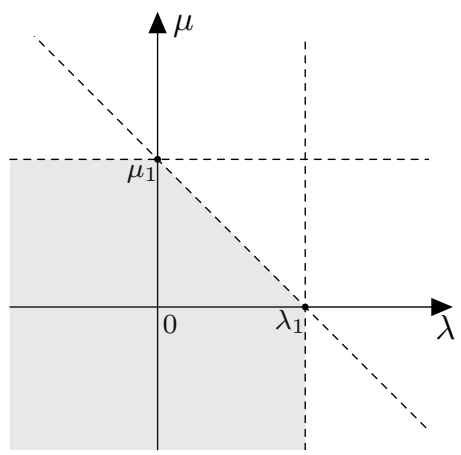

FIGURE 1.

In the next result, we study the case where the nonlinearity $g$ is between two consecutive eigenvalues $\mu_{j}$, while the other nonlinearity can be in resonance with one of the eigenvalues.

Theorem 1.3. Assume $(\mathbf{P}),(\mathbf{H 1}),(\mathbf{G 1}),(\mathbf{F} \mathbf{1}),(\mathbf{L G})$ and $(\mathbf{L F})$. In addition, suppose:

(A2) there exist constants $A, B, \alpha, \beta, M \in \mathbb{R}$ such that

$$
\mu_{j}<A \leq \liminf _{|U| \rightarrow+\infty} \frac{2 G(x, U)}{u^{2}+v^{2}} \leq \limsup _{|U| \rightarrow+\infty} \frac{2 G(x, U)}{u^{2}+v^{2}} \leq B<\mu_{j+1}
$$

and

$$
\alpha \leq \liminf _{|U| \rightarrow+\infty} \frac{2 F(x, U)}{u^{2}+v^{2}} \leq \limsup _{|U| \rightarrow+\infty} \frac{2 F(x, U)}{u^{2}+v^{2}} \leq \beta,
$$

uniformly for $x \in \bar{\Omega}$, with $\mu_{j} \lambda_{1}<\lambda_{1} A+\mu_{j} \alpha$ and $\lambda_{1} B+\mu_{j+1} \beta<$ $\mu_{j+1} \lambda_{1}$.

(A3) There exists $M \in \mathbb{R}$ such that, for $U \in \mathbb{R}^{2}$,

$$
\begin{gathered}
f\left(x, U_{n}\right) \cdot U_{n}-2 F\left(x, U_{n}\right) \geq M, \\
\text { uniformly almost everywhere } x \in \Omega,
\end{gathered}
$$


and

$$
\begin{gathered}
g\left(x, U_{n}\right) \cdot U_{n}-2 G\left(x, U_{n}\right) \geq M, \\
\text { uniformly almost everywhere } x \in \partial \Omega .
\end{gathered}
$$

Then system (1.1) has at least one solution $U \in H(\Omega)$.

In Figure 2, the hatched region in the Cartesian plane $\lambda \mu$ illustrates

$$
\mu_{j} \lambda_{1}<\lambda_{1} A+\mu_{j} \alpha
$$

and

$$
\lambda_{1} B+\mu_{j+1} \beta<\mu_{j+1} \lambda_{1} .
$$

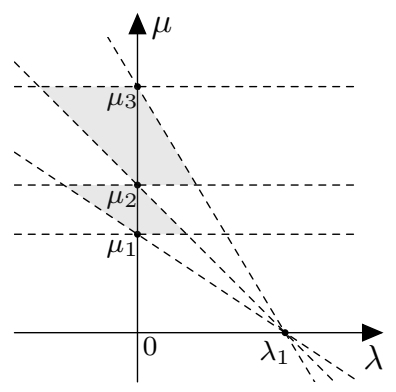

FIGURE 2.

The next result studies when the nonlinearity $f$ is between two consecutive eigenvalues $\lambda_{j}$, while the other nonlinearity can be in resonance with one of the eigenvalues.

Theorem 1.4. Suppose that the assumptions (P), (H1), (G1), (F1), (LG) and (LF) are satisfied, and the following conditions hold:

(A4) There exist constants $A, B, \alpha, \beta \in \mathbb{R}$ such that

$$
A \leq \liminf _{|U| \rightarrow+\infty} \frac{2 G(x, U)}{u^{2}+v^{2}} \leq \limsup _{|U| \rightarrow+\infty} \frac{2 G(x, U)}{u^{2}+v^{2}} \leq B
$$

and

$$
\lambda_{j}<\alpha \leq \liminf _{|U| \rightarrow+\infty} \frac{2 F(x, U)}{u^{2}+v^{2}} \leq \limsup _{|U| \rightarrow+\infty} \frac{2 F(x, U)}{u^{2}+v^{2}} \leq \beta<\lambda_{j+1},
$$


uniformly for $x \in \bar{\Omega}$, with

$$
\lambda_{j} \mu_{1}<\lambda_{j} A+\mu_{1} \alpha
$$

and

$$
\lambda_{j+1} B+\mu_{1} \beta<\lambda_{j+1} \mu_{1} .
$$

Then system (1.1) admits at least one solution $U \in H(\Omega)$.

In Figure 3, the hatched region in the Cartesian plane $\lambda \mu$ illustrates

$$
\lambda_{j} \mu_{1}<\lambda_{j} A+\mu_{1} \alpha
$$

and

$$
\lambda_{j+1} B+\mu_{1} \beta<\lambda_{j+1} \mu_{1} .
$$

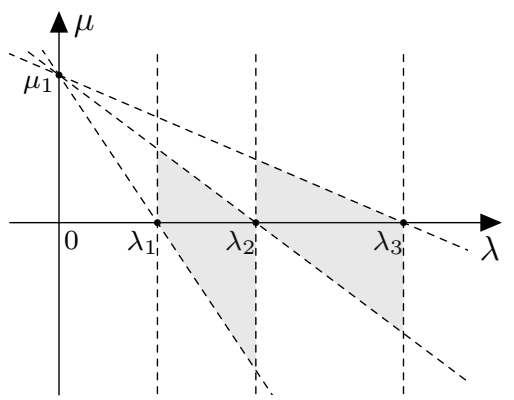

FIGURE 3.

The above results extend the results in [17], which were established in the scalar case.

2. Preliminaries. The proof is accomplished by variational arguments. So, we define the functional associated to system (1.1),

$$
I: H(\Omega) \longrightarrow \mathbb{R},
$$

given by

$$
\begin{aligned}
I(U)= & \frac{1}{2} \int_{\Omega}\left[|\nabla U|^{2}+\langle C(x) U, U\rangle\right] d x \\
& -\int_{\Omega} F(x, U) d x-\int_{\partial \Omega} G(x, U) d \sigma .
\end{aligned}
$$


From $(\mathbf{P}),(\mathbf{H 1}),(\mathbf{G 1})$ and $(\mathbf{F 1})$, the functional $I \in C^{1}(H(\Omega), \mathbb{R})$ and its Fréchét derivative in $U \in H(\Omega)$ is given by

$$
\begin{aligned}
I^{\prime}(U) V=\langle U, V\rangle_{C}- & \int_{\Omega} f(x, U) \cdot V d x-\int_{\partial \Omega} g(x, U) \cdot V d \sigma, \\
& \text { for all } V \in H(\Omega) .
\end{aligned}
$$

Proposition 2.1. Suppose (P), (H1), (G1) and (F1) hold. Split the functional $I$ into $I=J_{1}-J_{2}-J_{3}$. Then the functionals

$$
J_{i}: H(\Omega) \longrightarrow \mathbb{R}, \quad \text { for } i=2,3,
$$

are weakly continuous. In addition, the operators $J_{2}^{\prime}$ and $J_{3}^{\prime}$ are compact.

Proof.

Claim. $J_{3}$ is weakly continuous. Indeed, let $\left(U_{m}=\left(u_{m}, v_{m}\right)\right)$ be a sequence in $H(\Omega)$ and $U=(u, v) \in H(\Omega)$, such that

$$
U_{m} \rightarrow U \text { weakly in }\left(H(\Omega),\|\cdot\|_{C}\right) .
$$

Since the embedding $H^{1}(\Omega)$ into $L^{s+1}(\partial \Omega)$ is compact for $1 \leq s+1<$ $(2 N-2) /(N-2)$, we have, up to a subsequence,

$$
U_{m} \longrightarrow U \quad \text { in } \quad\left(\left[L^{s+1}(\partial \Omega)\right]^{2},\|\cdot\|_{s+1}\right) .
$$

By the mean value theorem, there exists $\theta \in(0,1)$, such that

$$
\left|J_{3}\left(U_{m}\right)-J_{3}(U)\right|=\left|\int_{\partial \Omega} g\left(x, \theta U_{m}+(1-\theta) U\right) \cdot\left(U_{m}-U\right) d \sigma\right| .
$$

Applying the Hölder inequality, we obtain

$$
\left|J_{3}\left(U_{m}\right)-J_{3}(U)\right| \leq\left\|g\left(x, \Gamma_{m}(U)\right)\right\|_{(s+1) / s}\left\|U_{m}-U\right\|_{s+1},
$$

where $\Gamma_{m}(U)=\theta U_{m}+(1-\theta) U$. This verifies our claim, since

$$
\left\|g\left(x, \Gamma_{m}(U)\right)\right\|_{(s+1) / s}
$$

is bounded in $\mathbb{R}$.

Claim. $J_{2}$ is weakly continuous. The proof is similar to that above. 
Claim. $J_{3}^{\prime}$ is a compact operator. In fact, let $\left(U_{m}\right)$ be a bounded sequence in $\left(H(\Omega),\|\cdot\|_{C}\right)$. Then, up to a subsequence, we have

$$
U_{m} \longrightarrow U \quad \text { in }\left(\left[L^{s+1}(\partial \Omega)\right]^{2},\|\cdot\|_{s+1}\right) ;
$$

therefore,

$$
\left\|g\left(x, U_{m}\right)-g(x, U)\right\|_{(s+1) / s} \longrightarrow 0,
$$

as $m \rightarrow \infty$. On the other hand,

$$
\begin{aligned}
\left\|J_{3}^{\prime}\left(U_{m}\right)-J_{3}^{\prime}(U)\right\|_{C}^{*} & \leq \sup _{\|V\| \leq 1} \int_{\partial \Omega}\left|g\left(x, U_{m}\right)-g(x, U)\right|\|V\| d \sigma \\
& \leq C\left\|g\left(x, U_{m}\right)-g(x, U)\right\|_{(s+1) / s}
\end{aligned}
$$

Combining the above facts, the claim is proven.

Claim. $J_{2}^{\prime}$ is a compact operator. The proof is similar to that above.

Definition 2.2. Let $(E,\|\cdot\|)$ be a Banach space and $J \in C^{1}(E, \mathbb{R})$. Then $J$ satisfies the Palais-Smale condition, (PS), if every sequence $\left(u_{m}\right)$ in $E$, is such that

(i) $\left(J\left(u_{m}\right)\right)$ is bounded,

(ii) $J^{\prime}\left(u_{m}\right) \rightarrow 0$ in $\left(E^{*},\|\cdot\|^{*}\right)$ admits a convergent subsequence in $E$.

The following proposition arises from the above results.

Proposition 2.3. Assume (P), (H1), (G1) and (F1). If $\left(U_{m}\right)$ is a bounded sequence in $\left(H(\Omega),\|\cdot\|_{C}\right)$ such that $I^{\prime}\left(U_{m}\right) \rightarrow 0$ in $\left(H(\Omega)^{*}\right.$, $\left.\|\cdot\|_{C}^{*}\right)$, then $\left(U_{m}\right)$ admits a convergent subsequence in $E$.

Next we define the (PS) condition type, called the Cerami condition, introduced in [10].

Definition 2.4. Let $(E,\|\cdot\|)$ be a Banach space and $J \in C^{1}(E, \mathbb{R})$. We say that $J$ satisfies the Cerami condition at level $c, c \in \mathbb{R},(C e)_{c}$, if, for every sequence $\left(u_{m}\right)$ in $E$ : 
(Ce-1) $J\left(u_{m}\right) \rightarrow c$ as $m \rightarrow+\infty$,

(Ce-2) $\left(1+\left\|u_{m}\right\|\right)\left\|J^{\prime}\left(u_{m}\right)\right\|^{*} \rightarrow 0$ as $m \rightarrow+\infty$

possess a convergent subsequence in $E$.

The next proposition gives a sufficient condition for the Cerami condition.

Proposition 2.5. Let $(E,\|\cdot\|)$ be a Banach space, $c \in \mathbb{R}$, and $J \in C^{1}(E, \mathbb{R})$ such that:

(I) Every bounded sequence $\left(u_{m}\right) \subset E$, such that $J\left(u_{m}\right) \rightarrow c$ in $(\mathbb{R},|\cdot|)$ and $J^{\prime}\left(u_{m}\right) \rightarrow 0$ in $\left(E^{*},\|\cdot\|^{*}\right)$, admits a convergent subsequence in $E$;

(II) There exist constants $\delta, R, \alpha>0$ such that $\left\|J^{\prime}(u)\right\|^{*}\|u\| \geq \alpha$ for all $u \in J^{-1}([c-\delta, c+\delta])$, with $\|u\| \geq R$.

Then J satisfies the Cerami condition at level $c$.

Proof. Let $\left(u_{m}\right)$ be a sequence in $E$ satisfying conditions (Ce-1) and (Ce-2). Notice that, either $\left\|u_{m}\right\| \rightarrow+\infty$ as $m \rightarrow+\infty$, or $\left(u_{m}\right)$ is bounded in $E$. However, if $\left\|u_{m}\right\| \rightarrow+\infty$ as $m \rightarrow+\infty$, then, we have for $R>0$ (from (II)), that there exists $N_{0} \in \mathbb{N}$ such that $\left\|u_{m}\right\| \geq R$ for all $m \geq N_{0}$. Moreover, by condition (Ce-1), we obtain $N_{1} \in \mathbb{N}$ with $N_{1} \geq N_{0}$ such that $u_{m} \in J^{-1}([c-\delta, c+\delta])$ for all $m \geq N_{1}$. Consequently, following from condition (II), $\left\|J^{\prime}\left(u_{m}\right)\right\|^{*}\left\|u_{m}\right\| \geq \alpha$, for all $m \geq N_{1}$. Hence, by using (Ce-2), we have for $m \rightarrow+\infty$ that

$$
\alpha \leq\left\|J^{\prime}\left(u_{m}\right)\right\|^{*}\left\|u_{m}\right\| \leq\left\|J^{\prime}\left(u_{m}\right)\right\|^{*}\left(1+\left\|u_{m}\right\|\right) \longrightarrow 0,
$$

so $\alpha \leq 0$, which contradicts condition (II). Therefore, we should have $\left(u_{n}\right)$ bounded in $E$.

Claim. $J^{\prime}\left(u_{m}\right) \rightarrow 0$ on $\left(E^{*},\|\cdot\|^{*}\right)$.

Indeed, suppose by contradiction that there are $\epsilon>0$ and a subsequence $\left(u_{m_{l}}\right)$ of the sequence $\left(u_{m}\right)$, such that

$$
\left\|J^{\prime}\left(u_{m_{l}}\right)\right\|^{*} \geq \epsilon, \quad \text { for all } l \in \mathbb{N} .
$$

Then, we obtain

$$
\epsilon\left(1+\left\|u_{m_{l}}\right\|\right) \leq\left\|J^{\prime}\left(u_{m_{l}}\right)\right\|^{*}\left(1+\left\|u_{m_{l}}\right\|\right), \quad \text { for all } l \in \mathbb{N} .
$$


But, since $\left(u_{m}\right)$ is bounded in $E$, we have $\left(\left\|u_{m_{l}}\right\|\right)$ bounded in $\mathbb{R}$, and thus, we can consider $\left(\left\|u_{m_{l}}\right\|\right)$ convergent in $\mathbb{R}$, that is, there are $K_{1} \geq 0$ such that $\left\|u_{m_{l}}\right\| \rightarrow K_{1}$ as $l \rightarrow+\infty$. Then, passing the limit $l \rightarrow+\infty$ in (2.1) and using condition (Ce-2), $K_{1} \epsilon+\epsilon \leq 0$, which is absurd. Thus, we conclude the proof of the claim.

Now, from this claim and since $\left(u_{n}\right)$ is bounded in $E$ and (Ce-1) holds, we obtain from $(\mathbf{I})$ that $\left(u_{m}\right)$ has a convergent subsequence $E$.

We apply an abstract minimax theorem found in [13, Theorem 7], which is an extended version of the saddle point theorem due to $[\mathbf{1 8}$, Theorem 4.6]).

Proposition 2.6. Let $(E,\|\cdot\|)$ be a Banach space and $J \in C^{1}(E, \mathbb{R})$. Suppose that $E=\mathbb{V} \oplus \mathbb{X}$ with $\operatorname{dim} \mathbb{V}<\infty$. There exists an $R>0$ such that

$$
\sup _{u \in \partial \mathbb{D}} J(u) \leq \inf _{u \in \mathbb{X}} J(u)
$$

and $J$ satisfies the Cerami condition $(C e)_{c}$ at level

$$
c=\inf _{\gamma \in \mathbb{H}} \max _{u \in \mathbb{D}} J(\gamma(u)),
$$

with

$$
\begin{aligned}
& \mathbb{H}=\left\{\gamma \in C(\mathbb{D}, E):\left.\gamma\right|_{\partial \mathbb{D}}=i d\right\}, \\
& \mathbb{D}=\{u \in \mathbb{V}:\|u\| \leq R\},
\end{aligned}
$$

and

$$
\partial \mathbb{D}=\{u \in \mathbb{V}:\|u\|=R\} .
$$

Then $c \geq \inf _{u \in \mathbb{X}} J(u)$ and $c$ is a critical value of $J$.

The following classic abstract result can be found in $[\mathbf{1 8}, \mathbf{1 9}]$.

Proposition 2.7. Let $(E,\|\cdot\|)$ be a Banach space. If $I \in C^{1}(E, \mathbb{R})$ is bounded below and satisfies the (PS) condition, then $c \doteq \inf _{E} I$ is a critical value of $I$. 
3. Proof of Theorem 1.2. Recalling that $U=(u, v)$, from (A1) and given $\epsilon>0$, there exists a positive constant $M_{\epsilon}>0$ such that

$$
\begin{gathered}
G(x, U) \leq \frac{1}{2}(\mu+\epsilon)\left(u^{2}+v^{2}\right)+M_{\epsilon}, \\
\quad \text { for all } x \in \bar{\Omega}, \quad \text { for all } U \in \mathbb{R}^{2},
\end{gathered}
$$

and

$$
\begin{gathered}
F(x, U) \leq \frac{1}{2}(\lambda+\epsilon)\left(u^{2}+v^{2}\right)+M_{\epsilon}, \\
\quad \text { for all } x \in \bar{\Omega}, \quad \text { for all } U \in \mathbb{R}^{2} .
\end{gathered}
$$

We shall check the assumptions of Proposition 2.7. Since we already know that $I \in C^{1}(H(\Omega), \mathbb{R})$, it suffices to show the following claims.

Claim 1. I is coercive on $\left(H(\Omega),\|\cdot\|_{C}\right)$, that is, $I(U) \rightarrow+\infty$ as $\|U\|_{C} \rightarrow+\infty$.

Claim 2. I is bounded from below.

Claim 3. I satisfies the Palais-Smale (PS) condition.

Verification of Claim 1. Suppose that $\|U\|_{C} \rightarrow+\infty$. By continuity of the embedding $H(\Omega) \subset L^{2}(\partial \Omega)$ and equivalence of the norms $\|\cdot\|_{C}$ and $\|\cdot\|_{H}$, we infer that either $\|U\|_{2, \partial} \rightarrow+\infty$ or $\|U\|_{2, \partial} \leq K_{1}$, where $K_{1}>0$ is a constant. First, suppose that $\|U\|_{2, \partial} \leq K_{1}$. From equations (3.1) and (3.2), we have

$$
\begin{aligned}
I(U)= & \frac{1}{2}\|U\|_{C}^{2}-\int_{\Omega} F(x, U) d x-\int_{\partial \Omega} G(x, U) d \sigma \\
\geq & \frac{1}{2}\|U\|_{C}^{2}-\frac{1}{2}(\lambda+\epsilon)\|U\|_{2}^{2} \\
& -\frac{1}{2}(\mu+\epsilon)\|U\|_{2, \partial}^{2}-M_{\epsilon}\left(|\Omega|+|\partial \Omega|_{\sigma}\right) .
\end{aligned}
$$

If $\lambda<0$, then by (3.3), we conclude that $I(U) \rightarrow+\infty$ as $\|U\|_{C} \rightarrow+\infty$, taking $\epsilon>0$ such that $\lambda+\epsilon<0$. 
If $\lambda \geq 0$, from inequalities (1.3) and (3.3) with $\epsilon>0$ such that $\lambda+\epsilon>0$, we obtain

$$
I(U) \geq \frac{1}{2}\left(1-\frac{\lambda}{\lambda_{1}}-\frac{\epsilon}{\lambda_{1}}\right)\|U\|_{C}^{2}-\frac{1}{2}(\mu+\epsilon)\|U\|_{2, \partial}^{2}-M_{\epsilon}\left(|\Omega|+|\partial \Omega|_{\sigma}\right) .
$$

Since $\lambda<\lambda_{1}$, choose $\epsilon>0$, such that

$$
1-\frac{\lambda}{\lambda_{1}}-\frac{\epsilon}{\lambda_{1}}>0, \quad \lambda \geq 0 .
$$

By equation (3.4), we conclude that $I(U) \rightarrow+\infty$ as $\|U\|_{C} \rightarrow+\infty$.

Suppose now that $\|U\|_{2, \partial} \rightarrow+\infty$. We have four cases:

Case 1. $\lambda<0$ and $\mu<0$. From equation (3.3), choosing $\epsilon>0$ such that $\lambda+\epsilon>0$ and $\mu+\epsilon>0$, we obtain

$$
I(U) \geq \frac{1}{2}\|U\|_{C}^{2}-M_{\epsilon}\left(|\Omega|+|\partial \Omega|_{\sigma}\right),
$$

from which it follows that $I(U) \rightarrow+\infty$ as $\|U\|_{C} \rightarrow+\infty$.

Case 2. $\lambda<0$ and $\mu \geq 0$. Using equations (1.2) and (3.3) for $\epsilon>0$ such that $\mu+\epsilon>0$, we get

$$
I(U) \geq \frac{1}{2}\left(1-\frac{\mu}{\mu_{1}}-\frac{\epsilon}{\mu_{1}}\right)\|U\|_{C}^{2}-M_{\epsilon}\left(|\Omega|+|\partial \Omega|_{\sigma}\right) .
$$

Since $\mu<\mu_{1}$, by choosing $\epsilon>0$ such that

$$
1-\frac{\mu}{\mu_{1}}-\frac{\epsilon}{\mu_{1}}>0, \quad \mu \geq 0,
$$

we conclude that $I(U) \rightarrow+\infty$ as $\|U\|_{C} \rightarrow+\infty$.

Case 3. $\lambda \geq 0$ and $\mu<0$. By assumption, $\lambda<\lambda_{1}$, choose $\epsilon>0$ such that

$$
1-\frac{\lambda}{\lambda_{1}}-\frac{\epsilon}{\lambda_{1}}>0 .
$$

From equations (1.3) and (3.3), we have

$$
I(U) \geq \frac{1}{2}\left(1-\frac{\lambda}{\lambda_{1}}-\frac{\epsilon}{\lambda_{1}}\right)\|U\|_{C}^{2}-M_{\epsilon}\left(|\Omega|+|\partial \Omega|_{\sigma}\right) .
$$

Consequently, if $\|U\|_{C} \rightarrow+\infty$, we get $I(U) \rightarrow+\infty$. 
Case 4. $\lambda \geq 0$ and $\mu \geq 0$. By equations (1.3) and (3.3), we obtain

$$
I(U) \geq \frac{1}{2}\left(1-\frac{\lambda}{\lambda_{1}}-\frac{\epsilon}{\lambda_{1}}\right)\|U\|_{C}^{2}-\frac{1}{2}(\mu+\epsilon)\|U\|_{2, \partial}^{2}-C(\epsilon),
$$

since $\lambda+\epsilon>0$ and $\mu+\epsilon>0$, where $C(\epsilon) \doteq M_{\epsilon}\left(|\Omega|+|\partial \Omega|_{\sigma}\right)$ is a positive constant. By hypothesis, $\lambda<\lambda_{1}$, for $\epsilon>0$, we have

$$
1-\frac{\lambda}{\lambda_{1}}-\frac{\epsilon}{\lambda_{1}}>0
$$

and, using equations (1.2) and (3.5), we infer that

$$
I(U) \geq \frac{\mu_{1}}{2}\left[\left(1-\frac{\lambda}{\lambda_{1}}-\frac{\mu}{\mu_{1}}\right)-\epsilon\left(\frac{1}{\lambda_{1}}+\frac{1}{\mu_{1}}\right)\right]\|U\|_{2, \partial}^{2}-C(\epsilon) .
$$

By hypothesis, $\lambda \mu_{1}+\mu \lambda_{1}<\lambda_{1} \mu_{1}$. Then, choosing $\epsilon>0$ such that

$$
\left(1-\frac{\lambda}{\lambda_{1}}-\frac{\mu}{\mu_{1}}\right)-\epsilon\left(\frac{1}{\lambda_{1}}+\frac{1}{\mu_{1}}\right)>0,
$$

and by using equation (3.6), we conclude that $I(U) \rightarrow+\infty$ as $\|U\|_{C} \rightarrow$ $+\infty$ (supposing $\|U\|_{2, \partial} \rightarrow+\infty$ ).

Verification of Claim 2. From Claim 1, there exists $R_{1}>0$ such that

$$
I(U) \geq 1, \quad \text { for all } U \in H(\Omega), \text { with }\|U\|_{C} \geq R_{1} .
$$

Now, for $U \in H(\Omega)$ such that $\|U\|_{C} \leq R_{1}$, since $\|\cdot\|_{C}$ and $\|\cdot\|_{H}$ are equivalent norms in $H(\Omega)$, there exists a constant $R_{2}>0$, such that $\|U\|_{2} \leq R_{2}$ and $\|U\|_{2, \partial} \leq R_{2}$. Using equation (3.3) and recalling that $\lambda<\lambda_{1}$ and $\mu<\mu_{1}$, we infer that, for $\epsilon>0$ fixed,

$$
I(U) \geq-\frac{1}{2}\left(\lambda_{1}+\epsilon\right) R_{2}^{2}-\frac{1}{2}\left(\mu_{1}+\epsilon\right) R_{2}^{2}-M_{\epsilon}\left(|\Omega|+|\partial \Omega|_{\sigma}\right) \doteq K(\epsilon),
$$

that is,

$$
I(U) \geq K(\epsilon), \quad \text { for all } U \in H(\Omega) \text {, with }\|U\|_{C} \leq R_{1} .
$$

Combining inequalities (3.7) and (3.8), it follows that $I$ is bounded from below.

Verification of Claim 3. Let $\left(U_{m}\right)$ be a sequence in $\left(H(\Omega),\|\cdot\|_{C}\right)$ such that $\left(I\left(U_{m}\right)\right)$ is bounded in $\mathbb{R}$ and $I^{\prime}\left(U_{m}\right) \rightarrow 0$ in $\left(H(\Omega)^{*},\|\cdot\|_{C}^{*}\right)$ as $m \rightarrow \infty$. 
We claim that $\left(U_{m}\right)$ is a bounded sequence in $\left(H(\Omega),\|\cdot\|_{C}\right)$. Indeed, suppose on the contrary that, up to a subsequence, $\left\|U_{m}\right\|_{C} \rightarrow+\infty$, as $m \rightarrow+\infty$. By coercivity of the functional $I$, we get $I\left(U_{m}\right) \rightarrow$ $+\infty$, as $m \rightarrow+\infty$, which is a contradiction, because $\left(I\left(U_{m}\right)\right)$ is bounded $\mathbb{R}$. Therefore, $\left(U_{m}\right)$ is bounded in $\left(H(\Omega),\|\cdot\|_{C}\right)$. Finally by Proposition 2.3, the sequence $\left(U_{m}\right)$ admits a convergent subsequence in $\left(H(\Omega),\|\cdot\|_{C}\right)$. This means that $I$ satisfies the Palais-Smale condition.

Now, applying Theorem 2.7, we conclude that $I$ possesses at least a critical point $U \in H(\Omega)$, that is, $I^{\prime}(U)=0$. This means that $U$ is a weak solution for system (1.1).

4. Proof of Theorem 1.3. Now we will check the assumptions of Proposition 2.6. Since we already know that $I \in C^{1}(H(\Omega), \mathbb{R})$, it suffices to show the following lemmata and claims.

Lemma 4.1. Assume the hypotheses of Theorem 1.3. Suppose that there exist $c \in \mathbb{R}$ and a sequence $\left(U_{n}\right)$ in $H(\Omega)$ such that

$$
\left\|U_{n}\right\|_{C} \longrightarrow+\infty, \quad I\left(U_{n}\right) \longrightarrow c
$$

and

$$
\left\|I^{\prime}\left(U_{n}\right)\right\|_{C}^{*}\left\|U_{n}\right\| \longrightarrow 0 \text { as } n \rightarrow+\infty .
$$

Then there exists either $\Omega_{0} \subset \Omega$ with $\left|\Omega_{0}\right|>0$ such that $\left|U_{n}(x)\right| \rightarrow+\infty$ as $n \rightarrow+\infty$ almost everywhere $x \in \Omega_{0}$ or $\Omega_{1} \subset \partial \Omega$ with $\left|\Omega_{1}\right|_{\sigma}>0$ such that $\left|U_{n}(x)\right| \rightarrow+\infty$ as $n \rightarrow+\infty$ almost everywhere $x \in \Omega_{1}$.

Proof. By continuity and (A2), for $\epsilon>0$ there exist $C_{\epsilon}, E_{\epsilon}>0$ such that

$$
\begin{gathered}
G(x, U) \leq \frac{1}{2}(B+\epsilon)\left(u^{2}+v^{2}\right)+C_{\epsilon}, \\
\text { for all } x \in \bar{\Omega}, \text { for all } U \in \mathbb{R}^{2},
\end{gathered}
$$

and

$$
\begin{gathered}
F(x, U) \leq \frac{1}{2}(\beta+\epsilon)\left(u^{2}+v^{2}\right)+E_{\epsilon} \\
\text { for all } x \in \bar{\Omega}, \text { for all } U \in \mathbb{R}^{2} . \\
\widehat{U}_{n}=\frac{U_{n}}{\left\|U_{n}\right\|_{C}}
\end{gathered}
$$


Then, passing to a subsequence if necessary, there exists $U \in H(\Omega)$ such that $\widehat{U}_{n} \rightarrow \widehat{U}$ weakly in $\left(H(\Omega),\|\cdot\|_{H}\right)$. Then, we can assume

$$
\widehat{U}_{n} \rightarrow \widehat{U}, \quad\left(\left[L^{2}(\Omega)\right]^{2},\|\cdot\|_{2}\right) \quad \text { and } \quad\left(\left[L^{2}(\partial \Omega)\right]^{2},\|\cdot\|_{2, \partial}\right) .
$$

This implies that

$$
\widehat{U}_{n}(x) \longrightarrow \widehat{U}(x), \quad \text { as } n \rightarrow+\infty, \text { almost everywhere } x \in \Omega,
$$

and

(4.4) $\widehat{U}_{n}(x) \longrightarrow \widehat{U}(x)$, as $n \rightarrow+\infty$, almost everywhere $x \in \partial \Omega$.

By hypothesis, $I\left(U_{n}\right) \rightarrow c$ as $n \rightarrow+\infty$. Using inequalities (1.2), (1.3), (4.1) and (4.2), for $n$ sufficiently large, we obtain

$$
\begin{aligned}
\frac{1}{2}\left\|U_{n}\right\|_{C}^{2} \leq & (c+1)+\int_{\Omega} F\left(x, U_{n}\right) d x+\int_{\partial \Omega} G\left(x, U_{n}\right) d \sigma \\
\leq(c+1)+ & \frac{\beta}{2}\left\|U_{n}\right\|_{2}^{2}+\frac{B}{2}\left\|U_{n}\right\|_{2, \partial}^{2} \\
& +E_{\epsilon}|\Omega|+C_{\epsilon}|\partial \Omega|_{\sigma}+K_{n}(\epsilon),
\end{aligned}
$$

where

$$
K_{n}(\epsilon)=\frac{\epsilon}{2}\left[\lambda_{1}^{-1}\left\|U_{n}\right\|_{C}^{2}+\mu_{1}^{-1}\left\|U_{n}\right\|_{C}^{2}\right] .
$$

Now, multiplying (4.5) by $\left\|U_{n}\right\|_{C}^{-2}$, we obtain

$$
\frac{1}{2} \leq \frac{c+\epsilon}{\left\|U_{n}\right\|_{C}^{2}}+\frac{\beta}{2}\left\|\widehat{U}_{n}\right\|_{2}^{2}+\frac{B}{2}\left\|\widehat{U}_{n}\right\|_{2, \partial}^{2}+\frac{\epsilon}{2}\left[\lambda_{1}^{-1}+\mu_{1}^{-1}\right] .
$$

Passing to the limit in (4.6), we infer that

$$
\frac{1}{2} \leq \frac{\beta}{2}\|\widehat{U}\|_{2}^{2}+\frac{B}{2}\|\widehat{U}\|_{2, \partial}^{2},
$$

which implies that either $\left|\Omega_{0}\right|>0$ or $\left|\Omega_{1}\right|_{\sigma}>0$, where

$$
\Omega_{0}=\{x \in \Omega: \widehat{U}(x) \neq 0\}
$$

and

$$
\Omega_{1}=\{x \in \partial \Omega: \widehat{U}(x) \neq 0\} .
$$

Combining the above with eqautions (4.3) and (4.4), we conclude for $n$ large enough, either $\widehat{U}_{n}(x) \neq 0$ almost everywhere $x \in \Omega_{0}$ or $\widehat{U}_{n}(x) \neq 0$ almost everywhere $x \in \partial \Omega$. 
Finally, since $U_{n}=\left\|U_{n}\right\|_{C} \widehat{U}_{n}$, we have either, as $n \rightarrow+\infty$ and $\left|U_{n}(x)\right| \rightarrow+\infty$ almost everywhere $x \in \Omega_{0}$ or $\left|U_{n}(x)\right| \rightarrow+\infty$ almost everywhere $x \in \Omega_{1}$. This proves Lemma 4.1.

Next, we will prove a compactness condition.

Lemma 4.2. Assume all the hypotheses of Theorem 1.3. Then I satisfies the Cerami condition $(\mathrm{Ce})_{c}$ for all $c \in \mathbb{R}$.

Proof. We shall check conditions (I) and (II) of Proposition 2.5. The first condition (I) is verified by Proposition 2.3, while for condition (II), suppose by contradiction, that there exist $c \in \mathbb{R}$ and a sequence $\left(U_{n}\right)$ in $H(\Omega)$ such that

$$
\left\|U_{n}\right\|_{c} \longrightarrow+\infty, \quad I\left(U_{n}\right) \longrightarrow c
$$

and

$$
\left\|I^{\prime}\left(U_{n}\right)\right\|_{C}^{*}\left\|U_{n}\right\| \longrightarrow 0, \quad \text { as } n \rightarrow+\infty .
$$

By hypothesis, we have

$$
\begin{aligned}
2 I\left(U_{n}\right)-I^{\prime}\left(U_{n}\right)\left(U_{n}\right)= & \int_{\Omega}\left[f\left(x, U_{n}\right)-2 F\left(x, U_{n}\right)\right] d x \\
& +\int_{\partial \Omega}\left[g\left(x, U_{n}\right)-2 G\left(x, U_{n}\right)\right] d \sigma .
\end{aligned}
$$

Consequently, by (4.7),

$$
\begin{aligned}
\lim _{n \rightarrow+\infty}\left[\int _ { \Omega } \left[f\left(x, U_{n}\right)\right.\right. & \left.-2 F\left(x, U_{n}\right)\right] d x \\
& \left.+\int_{\partial \Omega}\left[g\left(x, U_{n}\right)-2 G\left(x, U_{n}\right)\right] d \sigma\right]=2 c .
\end{aligned}
$$

By Lemma 4.1, there exists either $\Omega_{0} \subset \Omega$ with $\left|\Omega_{0}\right|>0$ such that $\left|U_{n}(x)\right| \rightarrow+\infty$ as $n \rightarrow+\infty$ almost everywhere $x \in \Omega_{0}$ or $\Omega_{1} \subset \partial \Omega$ with $\left|\Omega_{1}\right|_{\sigma}>0$ such that $\left|U_{n}(x)\right| \rightarrow+\infty$ as $n \rightarrow+\infty$ almost everywhere $x \in \Omega_{1}$. Combining this result with conditions (LF) and (LG), we obtain

$$
\lim _{n \rightarrow+\infty}\left[f\left(x, U_{n}(x)\right) \cdot U_{n}(x)-2 F\left(x, U_{n}(x)\right)\right]=+\infty,
$$


almost everywhere $x \in \Omega_{0}$, or

$$
\lim _{n \rightarrow+\infty}\left[g\left(x, U_{n}(x)\right) \cdot U_{n}(x)-2 G\left(x, U_{n}\right)(x)\right]=+\infty
$$

almost everywhere $x \in \Omega_{1}$.

Denote $P_{n}(x)=f\left(x, U_{n}(x)\right) \cdot U_{n}(x)-2 F\left(x, U_{n}(x)\right)$ and $Q_{n}(x)=$ $g\left(x, U_{n}(x)\right) \cdot U_{n}(x)-2 G\left(x, U_{n}(x)\right)$. If equation (4.9) occurs, applying Fatou's lemma and by (A3), we have

$$
\begin{aligned}
\liminf _{n \rightarrow+\infty}\left[\int_{\Omega} P_{n}(x) d x+\int_{\partial \Omega} Q_{n}(x) d \sigma\right] \\
\quad \geq \liminf _{n \rightarrow+\infty} \int_{\Omega_{0}} P_{n}(x) d x+M\left(\left|\Omega \backslash \Omega_{0}\right|+|\partial \Omega|_{\sigma}\right)=+\infty
\end{aligned}
$$

which is a contradiction to equation (4.8). Thus, equation (4.10) holds. Arguing as above, we have

$$
\begin{aligned}
\liminf _{n \rightarrow+\infty}\left[\int_{\Omega}\right. & \left.P_{n}(x) d x+\int_{\partial \Omega} Q_{n}(x) d \sigma\right] \\
& \geq \liminf _{n \rightarrow+\infty} \int_{\Omega_{1}} Q_{n}(x) d \sigma+M\left(|\Omega|+\left|\partial \Omega \backslash \Omega_{1}\right|_{\sigma}\right)=+\infty
\end{aligned}
$$

which contradicts equation (4.8). Therefore condition (II) of Proposition 2.5 holds. This proves Lemma 4.2.

Let

$$
\mathcal{M}_{k}=\left\{V_{1}^{k}, V_{2}^{k}, \ldots, V_{m_{k}}^{k}\right\} \subset H(\Omega) \cap\left[L^{2}(\Omega)\right]^{2}
$$

be the $C$-orthonormal basis of Steklov eigenfunctions associated to $\mu_{k}$. In [12], motivated in part by [4], it was proved that, for $j \in \mathbb{N}$ fixed,

$$
H(\Omega)=\mathbb{V}_{j} \oplus_{C} \mathbb{X}_{j}
$$

with $\operatorname{dim}\left(\mathbb{V}_{j}\right)<\infty$, where

$$
\begin{aligned}
& \mathbb{V}_{j}=\frac{\left[\bigcup_{k=1}^{j} \mathcal{M}_{k}\right],}{\left[\bigcup_{k=j+1}^{\infty} \mathcal{M}_{k}\right]}, \\
& \mathbb{Y}_{j}=
\end{aligned}
$$


and

$$
\mathbb{X}_{j}=\mathbb{Y}_{j} \oplus_{C}\left(H_{0}(\Omega)\right)^{2}
$$

Also, we have

$$
\|U\|_{2, \partial \Omega}^{2} \geq \mu_{j}^{-1}\|U\|_{C}^{2}, \quad \text { for all } U \in \mathbb{V}_{j},
$$

and

$$
\|U\|_{2, \partial \Omega}^{2} \leq \mu_{j+1}^{-1}\|U\|_{C}^{2}, \quad \text { for all } U \in \mathbb{Y}_{j}
$$

We claim the following.

Claim 4.3. There exists a constant $R>0$ such that

$$
\sup _{U \in \partial \mathbb{D}} I(U) \leq \inf _{V \in \mathbb{X}_{j}} I(V)
$$

with $\mathbb{D}=\left\{U \in \mathbb{V}_{j}:\|U\|_{C} \leq R\right\}$.

Now assuming the proof of Claim 4.3, by Lemmata 4.1 and 4.2 , we can apply Proposition 2.6 to conclude the proof of Theorem 1.3.

Proof of Claim 4.3. The proof will be accomplished in three steps.

Step 1. Firstly, we will prove that $-I$ is coercive on $\mathbb{V}_{j}$. Indeed, using (A2) together with continuity of the functions $G$ and $F$, given $\epsilon>0$, there exists a constant $\widetilde{C}>0$ such that, if $x \in \bar{\Omega}$ and $U \in \mathbb{R}^{2}$, then

$$
(A-\epsilon) \frac{\left(u^{2}+v^{2}\right)}{2}-\widetilde{C} \leq G(x, U) \leq(B+\epsilon) \frac{\left(u^{2}+v^{2}\right)}{2}+\widetilde{C}
$$

and

$$
(\alpha-\epsilon) \frac{\left(u^{2}+v^{2}\right)}{2}-\widetilde{C} \leq F(x, U) \leq(\beta+\epsilon) \frac{\left(u^{2}+v^{2}\right)}{2}+\widetilde{C} .
$$

Consequently, for $U=(u, v) \in \mathbb{V}_{j}$, we have

$$
\begin{aligned}
I(U) & =\frac{1}{2}\|U\|_{C}^{2}-\int_{\Omega} F(x, U) d x-\int_{\partial \Omega} G(x, U) d \sigma \\
& \leq \frac{1}{2}\|U\|_{C}^{2}+P(x, U)+Q(x, U)+\widehat{C},
\end{aligned}
$$


where $\widehat{C}$ is a positive constant,

$$
P(x, U)=-\frac{1}{2}(\alpha-\epsilon)\|U\|_{2}^{2}
$$

and

$$
Q(x, U)=-\frac{1}{2}(A-\epsilon)\|U\|_{2, \partial}^{2} \cdot
$$

Suppose, without loss of generality, that $\alpha \leq 0$ in (A2). Using (A2) and by inequalities (1.3) and (4.11), we have for $\epsilon>0$ with $A-\epsilon>0$,

$$
P(x, U) \leq-\frac{1}{2}(\alpha-\epsilon) \lambda_{1}^{-1}\|U\|_{C}^{2}
$$

and

$$
Q(x, U) \leq-\frac{1}{2}(A-\epsilon) \mu_{j}^{-1}\|U\|_{C}^{2}
$$

Inserting the above in (4.16), we obtain

$$
I(U) \leq \frac{1}{2}\left(1-\frac{\alpha}{\lambda_{1}}-\frac{A}{\mu_{j}}+\frac{\epsilon}{\lambda_{1}}+\frac{\epsilon}{\mu_{j}}\right)\|U\|_{C}^{2}+\widehat{C} .
$$

Finally, since $\lambda_{1} \mu_{j}<\alpha \mu_{j}+A \lambda_{1}, \mu_{j} \geq \mu_{1}>0$ and $\lambda_{1}>0$, choosing $\epsilon>0$ such that $A-\epsilon>0$ and

$$
1-\frac{\alpha}{\lambda_{1}}-\frac{A}{\mu_{j}}+\frac{\epsilon}{\lambda_{1}}+\frac{\epsilon}{\mu_{j}}<0,
$$

we conclude that $I(U) \rightarrow-\infty$ as $U \in \mathbb{V}_{j}$ and $\|U\|_{C} \rightarrow+\infty$. Hence, $-I$ is coercive on $\mathbb{V}_{j}$.

Step 2. Now, we will prove that $I$ is coercive on $\mathbb{X}_{j}$. In fact, for $U \in \mathbb{X}_{j}$, since $\mathbb{X}_{j}=\mathbb{Y}_{j} \oplus_{C} H_{0}(\Omega)$, there exist, in a unique way, $U^{0} \in H_{0}(\Omega)$ and $\bar{U} \in \mathbb{Y}_{j}$ such that $U=U^{0}+\bar{U}$. From (4.14) and (4.15) and recalling that $U^{0}$ and $\bar{U}$ are $C$-orthogonal, there exists a constant $\bar{C}>0$ such that

$$
\begin{aligned}
I(U) & =\frac{1}{2}\left\|U^{0}\right\|_{C}^{2}+\frac{1}{2}\|\bar{U}\|_{C}^{2}-\int_{\Omega} F(x, U) d x-\int_{\partial \Omega} G(x, U) d \sigma \\
& \geq \frac{1}{2}\left\|U^{0}\right\|_{C}^{2}+\frac{1}{2}\|\bar{U}\|_{C}^{2}-\frac{1}{2}(\beta+\epsilon)\|U\|_{2}^{2}-\frac{1}{2}(B+\epsilon)\|U\|_{2, \partial}^{2}-\bar{C} .
\end{aligned}
$$


Consider, without loss of generality, $\beta \geq 0$ in (A2). By (1.3), and noticing that $\|U\|_{2, \partial}=\|\bar{U}\|_{2, \partial}, U^{0}$ and $\bar{U}$ are $C$-orthogonal, we have

$$
\begin{aligned}
I(U) \geq & \frac{1}{2}\left(1-\frac{\beta}{\lambda_{1}}-\frac{\epsilon}{\lambda_{1}}\right)\left\|U^{0}\right\|_{C}^{2} \\
& +\frac{1}{2}\left[\left(1-\frac{\beta}{\lambda_{1}}-\frac{\epsilon}{\lambda_{1}}\right)\|\bar{U}\|_{C}^{2}-(B+\epsilon)\|\bar{U}\|_{2, \partial}^{2}\right]-\bar{C} .
\end{aligned}
$$

Combining this inequality with (4.12), we obtain

$$
\begin{aligned}
I(U) \geq & \frac{1}{2}\left(1-\frac{\beta}{\lambda_{1}}-\frac{\epsilon}{\lambda_{1}}\right)\left\|U^{0}\right\|_{C}^{2} \\
& +\frac{1}{2}\left(1-\frac{\beta}{\lambda_{1}}-\frac{B}{\mu_{j+1}}-\frac{\epsilon}{\lambda_{1}}-\frac{\epsilon}{\mu_{j+1}}\right)\|\bar{U}\|_{C}^{2}-\bar{C} .
\end{aligned}
$$

By hypotheses $\beta \mu_{j+1}+B \lambda_{1}<\lambda_{1} \mu_{j+1} \lambda_{1}>0$ and $B>\mu_{j}>0$, choose $\epsilon>0$ such that

$$
M(\epsilon) \doteq 1-\frac{\beta}{\lambda_{1}}-\frac{\epsilon}{\lambda_{1}}>0
$$

and

$$
N(\epsilon) \doteq 1-\frac{\beta}{\lambda_{1}}-\frac{B}{\mu_{j+1}}-\frac{\epsilon}{\lambda_{1}}-\frac{\epsilon}{\mu_{j+1}}>0 .
$$

From this, $\min \{M(\epsilon), N(\epsilon)\}>0$ and by (4.17), we have

$$
I(U) \geq \min \{M(\epsilon), N(\epsilon)\}\left(\left\|U^{0}\right\|_{C}^{2}+\|\bar{U}\|_{C}^{2}\right)=\min \{M(\epsilon), N(\epsilon)\}\|U\|_{C}^{2} .
$$

This implies that $I(U) \rightarrow+\infty$, when $U \in \mathbb{X}_{j}$ and $\|U\|_{C} \rightarrow+\infty$.

This proves Step 2.

Step 3. Conclusion. From Step 2, I is coercive on $\mathbb{X}_{j}$. Then there exists an $R_{1}>0$ such that $I(U) \geq 1$, for all $U \in \mathbb{X}_{j}$, with $\|U\|_{C}>R_{1}$. In addition, if $U \in \mathbb{X}_{j}$ satisfies $\|U\|_{C} \leq R_{1}$, arguing as in the proof of Step 2, we get

$$
I(U) \geq \min \left\{1,-R_{1}^{2}\right\}=-R_{1}^{2}, \quad \text { for all } U \in \mathbb{X}_{j} .
$$

By Step 1, taking $R_{2}<-R_{1}^{2}$, there exists an $R>0$ such that, for all $U \in \mathbb{V}_{j}$ with $\|U\|_{C} \geq R$, we have $I(U)<R_{2}<-R_{1}^{2}$. Consequently,

$$
\sup _{U \in \partial \mathbb{D}} I(U) \leq R_{2}<-R_{1}^{2}
$$


where $\mathbb{D}=\left\{U \in \mathbb{V}_{j}:\|U\|_{C} \leq R\right\}$. Combining these, the proof of Claim 4.3 is complete.

Now, applying Theorem 2.6, we conclude that $I$ has at least one critical point $U$, which is a weak solution of system (1.1). Hence, Theorem 1.3 is proven.

5. Proof of Theorem 1.4. We have already shown that $I \in$ $C^{1}(H(\Omega), \mathbb{R})$, and we can prove that $I$ verifies the Cerami condition $(\mathrm{Ce})_{c}$, arguing as in Theorem 1.3. Thus, it is enough to prove the geometry of Theorem 2.6.

The following result is proven in [12].

Let

$$
\mathcal{B}_{k}=\left\{U_{1}^{k}, U_{2}^{k}, \ldots, U_{\tau_{k}}^{k}\right\} \subset H(\Omega) \cap\left[L^{2}(\Omega)\right]^{2}
$$

be an orthonormal basis for the eigenspace associated to $\lambda_{k}$.

For each $j \in \mathbb{N}$ fixed, consider $\mathbb{F}_{j}$, the subspace generated by

$$
\mathbb{E}_{j}=\left\{U_{1}^{1}, \ldots, U_{\tau_{1}}^{1}, \ldots, U_{1}^{j}, \ldots, U_{\tau_{j}}^{j}\right\},
$$

which has dimension $\tau_{1}+\tau_{2}+\cdots+\tau_{j}$. Then

$$
\left[L^{2}(\Omega)\right]^{2}=\mathbb{F}_{j} \oplus \mathbb{F}_{j}^{\perp},
$$

where

$$
\mathbb{F}_{j}^{\perp}=\left\{U \in\left[L^{2}(\Omega)\right]^{2}:\langle U, V\rangle_{2}=0, \text { for all } V \in \mathbb{F}_{j}\right\} .
$$

Here, $\langle U, V\rangle_{2}=\int_{\Omega} U \cdot V d x$. Also, we obtain the decomposition

$$
H(\Omega)=\mathbb{F}_{j} \oplus\left[\mathbb{F}_{j}^{\perp} \cap H(\Omega)\right],
$$

where $\operatorname{dim} \mathbb{F}_{j}<\infty$ for $j \in \mathbb{N}$ fixed. In addition, we have the following inequalities:

$$
\|U\|_{2}^{2} \geq \lambda_{j}^{-1}\|U\|_{C}^{2}, \quad \text { for all } U \in \mathbb{F}_{j},
$$

and

$$
\|U\|_{2}^{2} \leq \lambda_{j+1}^{-1}\|U\|_{C}^{2}, \quad \text { for all } U \in\left[\mathbb{F}_{j}^{\perp} \cap H(\Omega)\right]
$$

Now, we need the following claim. 
Claim 5.1. There exists an $R>0$ such that

$$
\sup _{U \in \partial \mathbb{D}} I(U) \leq \inf _{V \in \mathbb{F}_{j}^{+} \cap H(\Omega)} I(V),
$$

where $\mathbb{D}=\left\{U \in \mathbb{F}_{j}:\|U\|_{C} \leq R\right\}$.

Assuming Claim 5.1, and applying Theorem 2.6, the proof of Theorem 1.4 is concluded.

The claim will be proved in three steps.

Proof. Step 1. The functional $-I$ is coercive on $\mathbb{F}_{j}$. Indeed, from (A4) and by continuity of $G$ and $F$, it follows that, given $\epsilon>0$, there exists a constant $\widetilde{C}>0$ such that, for $x \in \bar{\Omega}$ and $U \in \mathbb{R}^{2}$, we get

$$
(A-\epsilon) \frac{\left(u^{2}+v^{2}\right)}{2}-\widetilde{C} \leq G(x, U) \leq(B+\epsilon) \frac{\left(u^{2}+v^{2}\right)}{2}+\widetilde{C},
$$

and

$$
(\alpha-\epsilon) \frac{\left(u^{2}+v^{2}\right)}{2}-\widetilde{C} \leq F(x, U) \leq(\beta+\epsilon) \frac{\left(u^{2}+v^{2}\right)}{2}+\widetilde{C} .
$$

Using these estimates, for $U \in \mathbb{F}_{j}$, there exists a constant $\bar{C}>0$ such that

$$
\begin{aligned}
I(U) & =\frac{1}{2}\|U\|_{C}^{2}-\int_{\Omega} F(x, U) d x-\int_{\partial \Omega} G(x, U) d \sigma \\
& \leq \frac{1}{2}\|U\|_{C}^{2}+\widetilde{P}(x, U)+\widetilde{Q}(x, U)+\bar{C}
\end{aligned}
$$

where

$$
\widetilde{P}(x, U)=-\frac{1}{2}(\alpha-\epsilon)\|U\|_{2}^{2}
$$

and

$$
\widetilde{Q}(x, U)=-\frac{1}{2}(A-\epsilon)\|U\|_{2, \partial}^{2} .
$$

Therefore, assume without loss of generality that $A<0$ in (A4). Since $\alpha>\lambda_{j}>0$, from inequalities (1.2) and (5.1), for $\epsilon>0$, we get

$$
\widetilde{P}(x, U) \leq-\frac{1}{2}(\alpha-\epsilon) \lambda_{j}^{-1}\|U\|_{C}^{2}
$$


and

$$
\widetilde{Q}(x, U) \leq-\frac{1}{2}(A-\epsilon) \mu_{1}^{-1}\|U\|_{C}^{2} .
$$

From hypotheses $\lambda_{j} \mu_{1}<\lambda_{j} A+\mu_{1} \alpha, \lambda_{j} \geq \lambda_{1}>0$ and $\mu_{1}>0$, we can choose $\epsilon>0$ such that $\alpha-\epsilon>0$ and

$$
1-\frac{A}{\mu_{1}}-\frac{\alpha}{\lambda_{j}}+\frac{\epsilon}{\lambda_{j}}+\frac{\epsilon}{\mu_{1}}<0 .
$$

So, from equation (5.5), we infer that

$$
\begin{aligned}
I(U) & \leq \frac{1}{2}\|U\|_{C}^{2}-\frac{1}{2}(\alpha-\epsilon) \lambda_{j}^{-1}\|U\|_{C}^{2}-\frac{1}{2}(A-\epsilon) \mu_{1}^{-1}\|U\|_{C}^{2}+\bar{C} \\
& =\frac{1}{2}\left(1-\frac{A}{\mu_{1}}-\frac{\alpha}{\lambda_{j}}+\frac{\epsilon}{\lambda_{j}}+\frac{\epsilon}{\mu_{1}}\right)\|U\|_{C}^{2}+\bar{C} .
\end{aligned}
$$

Therefore, $I(U) \rightarrow-\infty$, as $U \in \mathbb{F}_{j}$ and $\|U\|_{C} \rightarrow+\infty$, that is, $-I(U) \rightarrow+\infty$, as $U \in \mathbb{F}_{j}$ and $\|U\|_{C} \rightarrow+\infty$. Hence, Step 1 is proven.

Step 2. The functional $I$ is coercive on $\mathbb{F}_{j}^{\perp} \cap H(\Omega)$. Indeed, for $U \in \mathbb{F}_{j}^{\perp} \cap H(\Omega)$, from equations (5.3) and (5.4), for $\epsilon>0$ and $x \in \bar{\Omega}$, there exists a constant $\widetilde{C}>0$ such that

$$
\begin{aligned}
I(U) & =\frac{1}{2}\|U\|_{C}^{2}-\int_{\Omega} F(x, U) d x-\int_{\partial \Omega} G(x, U) d \sigma \\
& \geq \frac{1}{2}\|U\|_{C}^{2}+P_{1}(x, U)+Q_{1}(x, U)+\widetilde{C},
\end{aligned}
$$

where $P_{1}(x, U)=-(\beta+\epsilon) / 2\|U\|_{2}^{2}$ and $Q_{1}(x, U)=-(B+\epsilon) / 2\|U\|_{2, \partial}^{2}$. Consequently, assume without loss of generality that $B \geq 0$ in (A4) and since $\beta>\lambda_{1}>0$ from equations (1.2) and (5.2), for $\epsilon>0$, we get

$$
P_{1}(x, U) \geq-\frac{1}{2} \lambda_{j+1}^{-1}(\beta+\epsilon)\|U\|_{C}^{2}
$$

and

$$
Q_{1}(x, U) \geq-\frac{1}{2} \mu_{1}^{-1}(B+\epsilon)\|U\|_{C}^{2}
$$

Finally, by hypothesis, $\lambda_{j+1} B+\mu_{1} \beta<\lambda_{j+1} \mu_{1}$ and choosing $\epsilon>0$ such that

$$
1-\frac{B}{\mu_{1}}-\frac{\beta}{\lambda_{j+1}}-\frac{\epsilon}{\mu_{1}}-\frac{\epsilon}{\lambda_{j+1}}>0,
$$


we conclude by using equation (5.6) that

$$
\begin{aligned}
I(U) & \geq \frac{1}{2}\|U\|_{C}^{2}-\frac{1}{2} \lambda_{j+1}^{-1}(\beta+\epsilon)\|U\|_{C}^{2}-\frac{1}{2} \mu_{1}^{-1}(B+\epsilon)\|U\|_{C}^{2}+\widetilde{C} \\
& =\frac{1}{2}\left(1-\frac{B}{\mu_{1}}-\frac{\beta}{\lambda_{j+1}}-\frac{\epsilon}{\mu_{1}}-\frac{\epsilon}{\lambda_{j+1}}\right)\|U\|_{C}^{2}+\widetilde{C} .
\end{aligned}
$$

Therefore taking $\|U\|_{C} \rightarrow+\infty$ with $U \in \mathbb{F}_{j}^{\perp} \cap H(\Omega)$, we obtain $I(U) \rightarrow+\infty$, that is, $I$ is coercive on $\mathbb{F}_{j}^{\perp} \cap H(\Omega)$.

Step 3. Here we present the conclusion of the prove of the claim. There exists an $R>0$ such that

$$
\sup _{U \in \partial \mathbb{D}} I(U) \leq \inf _{V \in \mathbb{F}_{j}^{\perp} \cap H(\Omega)} I(V),
$$

where $\mathbb{D}=\left\{U \in \mathbb{F}_{j}:\|U\|_{C} \leq R\right\}$.

Indeed, by Step 2, $I$ is coercive on $\mathbb{F}_{j}^{\perp} \cap H(\Omega)$. There exists an $R_{1}>0$ such that $I(U) \geq 1$ for all $U \in \mathbb{F}_{j}^{\perp} \cap H(\Omega)$, with $\|U\|_{C}>R_{1}$. Now, for $U \in \mathbb{F}_{j}^{\perp} \cap H(\Omega)$ satisfying $\|U\|_{C} \leq R_{1}$, then by equation (5.7), for $\epsilon>0$ such that

$$
1-\frac{B}{\mu_{1}}-\frac{\beta}{\lambda_{j+1}}-\frac{\epsilon}{\mu_{1}}-\frac{\epsilon}{\lambda_{j+1}}>0,
$$

we have

$$
I(U) \geq-\frac{1}{2}\left(1-\frac{B}{\mu_{1}}-\frac{\beta}{\lambda_{j+1}}-\frac{\epsilon}{\mu_{1}}-\frac{\epsilon}{\lambda_{j+1}}\right) R_{1}^{2} \doteq R_{2} .
$$

Consequently,

$$
\inf _{U \in \mathbb{F}_{j}^{\perp} \cap H(\Omega)} I(U) \geq R_{2} .
$$

By Step 1, given $R_{3}<R_{2}$, there exists an $R>0$ such that, for all $U \in \mathbb{F}_{j}$ with $\|U\|_{C} \geq R$, we get $I(U)<R_{3}<R_{2}$. This implies that

$$
\sup _{U \in \partial \mathbb{D}} I(U) \leq R_{3}<R_{2},
$$

where $\mathbb{D}=\left\{U \in \mathbb{F}_{j}:\|U\|_{C} \leq R\right\}$. Finally, combining inequalities (5.9) and (5.10), we have inequality (5.8). 
From Theorem 2.6, the functional $I$ possesses at least one critical point $U \in H(\Omega)$, which is a solution for system (1.1). This completes the proof of Theorem 1.4.

Acknowledgments. This paper was completed while the second author was visiting the Department of Mathematics of Rutgers University, whose hospitality he gratefully acknowledges. He would also like to express his gratitude to Prof. Haim Brezis.

\section{REFERENCES}

1. G.A. Afrouzi, S. Heidarkhani and D. O'Regan, Three solutions to a class of Neumann doubly eigenvalue elliptic ystems driven by a $\left(p_{1}, p_{2}, \ldots, p_{n}\right)$-Laplacian, Bull. Korean Math. Soc. 6 (2010), 1235-1250.

2. H. Amann, Maximum principles and principal eigenvalues, in Ten mathematical essays on approximation in analysis and topology, J. Ferrera, J. López-Gomez and F.R. Ruiz del Portal, eds., Elsevier, Amsterdam, The Netherlands, 2005.

3. G. Auchmuty, Bases and comparison results for linear elliptic eigenproblems, J. Math. Anal. Appl. 390 (2012), 394-406.

4. - Finite energy solutions of mixed elliptic boundary value problems, Math. Meth. Appl. Sci. 33 (2010), 1446-1462.

5. Steklov eigenproblems and the representation of solutions of elliptic boundary value problems, Numer. Funct. Anal. Optim. 25 (2004), 321-348.

6. Spectral characterization of the trace spaces $H^{s}(\partial \Omega)$, SIAM J. Math. Anal. 38 (2006), 894-905.

7. J.F. Bonder, S. Martinez and J.D. Rossi, Existence results for gradient elliptic systems with nonlinear boundary conditions, Nonlin. Diff. Equat. Appl. 14 (2007), 153-179.

8. K.J. Brown and T.F. Wu, A semilinear elliptic system involving nonlinear boundary condition and sign-changing weight function, J. Math. Anal. Appl. 337 (2008), 1326-1336.

9. D.G. Costa and C.A Magalhães, Variational elliptic problems which are nonquadratic at infinity, Nonlin. Anal. 23 (1994), 1401-1412.

10. G. Cerami, Un Criterio de Esistenza per i Punti Critici su Varietá Ilimitate, Rc. Ist. Lomb. Sci. Lett. 112 (1978), 332-336.

11. F.O. De Paiva, M.F. Furtado, Multiplicity of solutions for resonant elliptic systems, J. Math. Anal. Appl.319 (2006), 435-449.

12. J.D.B. Godoi, O.H. Miyagaki and R.S. Rodrigues, Steklov-Neumann eigenproblens: A spectral characterization of the Sobolev trace spaces, submitted.

13. N.C. Kourogenis and N.S. Papageorgiou, Nonsmooth critical point theory and nonlinear elliptic equations at resonance, J. Austral. Math. Soc. 69 (2000), $245-271$. 
14. P.D. Lamberti, Steklov-type eigenvalues associated with best Sobolev trace constants: domain perturbation and overdetermined systems, Complex Var. Ellip. Equat. 59 (2014), 309-323.

15. C. Li, The existence of infinitely many solutions of a class of nonlinear elliptic equations with Neumann boundary condition for both resonance and oscillation problems, Nonlin. Anal. 54 (2003), 441-443.

16. C. Li and $\mathrm{S} . \mathrm{Li}$, Multiple solutions and sign-changing solutions of a class of nonlinear elliptic equations with Neumann boundary condition, J. Math. Anal. Appl. 298 (2004), 14-32.

17. N. Mavinga and M.N. Nkashama, Steklov-Neumann eigenproblems and nonlinear elliptic equations with nonlinear boundary conditions, J. Differ. Equat. 248 (2010), 1212-1229.

18. P. Rabinowitz, Minimax methods in critical point theory with applications to differential equations, CBMS Reg. Conf. Ser. Math. 65, American Mathematical Society, Providence, RI, 1986.

19. M. Willem, Minimax theory, progress in nonlinear differential equations and their applications, Birkhäuser, Boston, 1996.

20. J. Zhang, S. Li, Y. Wang and X. Xue, Multiple solutions for semilinear elliptic equations with Neumann boundary condition and jumping nonlinearities, J. Math. Anal. Appl. 371 (2010), 682-690.

Universidade Federal de Santa Maria, Departamento de Matemática, CEP. 97105-900, SANTA MARIA, RS, BraziL

Email address: jdamiao7@yahoo.com.br

Universidade Federal de Juiz de Fora, Departamento de Matemática, CEP. 36036-330, Juiz dE ForA, MG, BRAzIL

Email address: ohmiyagaki@gmail.com

Universidade Federal de São Carlos, Departamento de Matemática, CEP. 13565-905, SÃo CARLOS, SP, BRAZIL

Email address: rodrigo@dm.ufscar.br 